Boletín de la Sociedad Zoológica del Uruguay, 2020

Vol. 29 (2): 141-149

ISSN 2393-6940

https://journal.szu.org.uy

\title{
LISTA PRELIMINAR DE LAS MARIPOSAS ACUÁTICAS Y SEMIACUÁTICAS (INSECTA: LEPIDOPTERA) PRESENTES EN EL URUGUAY
}

\author{
${ }^{1}$ Bentancur-Viglione, Gabriela; ${ }^{1}$ Castro, Manuel \& ${ }^{1}$ Morelli, Enrique \\ 1. Sección Entomología, Instituto de Biología, Facultad de Ciencias, UdelaR.
}

Autor para correspondencia: gbentancur@fcien.edu.uy

\section{RESUMEN}

El presente trabajo ofrece la primera lista de lepidópteros acuáticos y semiacuáticos registrados para el Uruguay. La misma está basada en datos bibliográficos, especímenes depositados en colección y registros recientes de relevamientos de campo. Comprende datos de 10 géneros (Paracles, Parapoynx, Petrophila, Argyractis, Samea, Nomophila, Neohelvibotys, Niphograpta, Nonagria y Coleophora) contenidos en cinco familias (Erebidae, Crambidae, Pyralidae, Noctuidae y Coleophoridae), se completa con un mapa de distribución geográfica para seis géneros y siete especies.

Palabras clave: lepidopterofauna acuática, bioindicadores, biodiversidad

\section{ABSTRACT \\ Preliminary list of aquatic and semi-aquatic moths (Insecta: Lepidoptera) present in Uruguay. The present work offers the first list of aquatic and semi-aquatic Lepidoptera registered for Uruguay. It is based on bibliographic data, specimens deposited in the collection and recent records from field surveys. It includes data from 10 genera (Paracles, Parapoynx, Petrophila, Argyractis, Samea, Nomophila, Neohelvibotys, Niphograpta, Nonagria y Coleophora) contained in five families (Erebidae, Crambidae, Pyralidae, Noctuidae y Coleophoridae). A complementary map of the geographical distribution for six genera and seven species is provided.}

Keywords: Aquatic Lepidoptera, bioindicators, biodiversity

\section{INTRODUCCIÓN}

El Orden Lepidoptera agrupa generalmente especies terrestres, pero algunas presentan estadios larvales asociados a ecosistemas acuáticos, e inclusive algunas son catalogadas como acuáticas estrictas.
Las especies semiacuáticas son aquellas que pasan solo una parte de su ciclo de vida en un ambiente acuático y cuyas larvas se alimentan y viven sobre o en el interior de vegetación acuática o emergente (Mey \& Speidel, 2008; Romero \& Navarro, 2009).

La mayor parte de los registros de estos lepidópteros se encuentra en las áreas tropicales (Romero \& Navarro, 2009) y para Sudamérica se registran ocho familias con estados larvales relacionados con ecosistemas de agua dulce (Lange, 1978; Domínguez \& Fernandez, 2001): Nepticulidae, Coleophoridae, Cosmopterigidae, Noctuidae, Tortricidae, Pyralidae, Crambidae y Erebidae (Romero \& Navarro, 2009; Morelli \& Bentancur-Viglione, 2013). Crambidae se destaca por el éxito adaptativo de sus larvas a la vida acuática, sobre todo los Nymphulinae con 233 especies y 22 géneros descritos con estas características (Klima, 1937; Lange, 1956; Munroe, 1995). Acentropinae, Pyraustinae (Crambidae) y Arctiinae (Erebidae) agrupan algunas de las pocas especies con larvas acuáticas estrictas, o sea que viven sumergidas durante todo su desarrollo (Mey \& Speidel, 2008).

En general en las especies de hábitos acuáticos, los imagos viven entre 24 horas y un mes dependiendo de la especie y del sexo (Lange, 1978). En muchas especies de vida corta las piezas bucales están atrofiadas y no son funcionales. La actividad reproductiva de estos insectos se desarrolla en general sobre la vegetación hospedera o sobre las piedras o sustratos emergentes (Lange, 1978; Buckingham \& Bennet, 2001). En algunos géneros de la familia Crambidae como Parapoynx (Hübner, [1825]) la cópula ocurre inmediatamente luego de la emergencia de la hembra cuando sus alas aún están mojadas. Luego de la cópula las hembras desovan utilizando distintas estrategias: los huevos pueden ser depositados en grupos de entre 10 a 400 en filas irregulares, entre grietas y tallos, en la base de piedras o sustratos sumergidos, en el envés de las hojas o sobre la superficie del agua (MacGaha, 1954; Maddox, 1970; Tuskes, 1977; Kinser \& Neunzing, 1981; Buckingham \& Bennet, 1996, 2001). 
Las adaptaciones larvales a la vida acuática tienen relación directa con el desarrollo de estrategias respiratorias sobre todo en aquellas larvas cuya ninfosis transcurre dentro de los propios cuerpos de agua. Así encontramos larvas donde el intercambio gaseoso se realiza a través del tegumento (larvas desnudas), por medio de sistemas de traqueobranquias torácicas y abdominales con distintos niveles de complejidad, así como el desarrollo de plastrones respiratorios en aquellas larvas que comparten ambientes sumergidos y semisumergidos (Welch, 1922; Romero \& Navarro, 2009).

Para Sudamérica los trabajos en sistemática larval son puntuales con descripciones para Brasil (Nessimian \& Da Silva, 1997; Meneses et al., 2013; Habeck \& Passoa, 1987), Paraguay (Drechsel, 2014; Drechsel \& Drechsel García, 2016a,b, 2017), Guayana (Sattler, 1961), Perú (Roback, 1966), Argentina (DeLoach et al., 1979; Berg, 1876a,b, 1877; Beccacece et al., 2014) y Bolivia (Munroe, 1974). Para Uruguay los primeros trabajos de lepidopterofauna acuática describen la biología y el desarrollo larval de Paracles azollae (Berg, 1877) autor y $P$. burmeisteri (Berg, 1877) (Berg, 1876a o b; 1877). A partir de 2012 comienza en Uruguay la identificación y caracterización de larvas de lepidópteros asociados a ecosistemas de agua dulce: Bentancur-Viglione \& Morelli, 2012, 2014, 2015; Morelli \& Bentancur-Viglione, 2012, 2013; BentancurViglione et al., 2016; Morelli et al., 2018a, b.

El objetivo del presente trabajo es presentar la diversidad de familias, géneros y especies de larvas acuáticas de lepidópteros que se han registrado en Uruguay. Además de citarse por primera vez para Uruguay el género Coleophora Hübner, 1822 (Coleophoridae). Se aportan datos de la distribución geográfica, y caracteres imaginales y larvales.

\section{MATERIALES Y MÉTODOS}

Se revisó la bibliografía para Uruguay y los países limítrofes, así como se inventariaron los ejemplares de imagos y larvas depositados en la Colección Entomológica de la Facultad de Ciencias, UdelaR, Montevideo, Uruguay. Se incorporaron datos de captura de campo de larvas e imagos en el período 2002-2019. La recolección de las larvas se realizó utilizando una red de mano de $300 \mu$ de luz de malla. El tiempo estandarizado de recolección fue de 10 minutos, sobre la vegetación acuática. Las larvas se sacrificaron en agua hirviendo y se fijaron en etanol $70 \%$. El material se depositó en la Colección de Entomología de la Facultad de Ciencias, Montevideo.

La identificación larval se realizó en base a las claves de Romero \& Navarro (2009) y Carter \& Kristensen (1998). La terminología larval fue la propuesta por Hinton (1946) con modificaciones de
Stehr (1987). Algunas larvas solo pudieron determinarse hasta el nivel de género, por no contar con claves específicas aún para lepidópteros acuáticos.

Para la identificación de las larvas de Paracles azollae (Berg, 1877) y $P$. vulpina (Hübner, [1825]) se criaron hasta la emergencia de los imagos. Estos ejemplares fueron extendidos y depositados en la colección de Entomología de la Facultad de Ciencias, junto con el material larval.

Para la elaboración del mapa de distribución se utilizó software QGIS 3.10, con el mismo se marcaron los registros puntuales georeferenciados, mientras que para las citas sin georeferenciar del material histórico depositado en colección y citas bibliográficas se procedió a marcar la ciudad o departamento mencionado. $\mathrm{E}$ inclusive algunos registros bibliográficos de especies de lepidópteros acuáticos solo mencionan el país (Uruguay), no pudiendo ilustrarse su distribución geográfica.

\section{RESULTADOS}

La evidencia disponible indica que en Uruguay se han registrado 10 géneros para cinco familias. Mientras doce especies han sido determinadas y citadas para el país como subacuáticas.

\section{Erebidae (Leach, [1815])}

Es una de las familias más grandes en número de especies y está compuesta por 18 subfamilias. Contempla especies que van desde $6 \mathrm{~mm}$ de expansión alar a otras de más de $250 \mathrm{~mm}$ (Zahiri, 2012). Larvas se caracterizan por presentar patas torácicas presentes, espuripedios presentes en número variable, pero siempre en A5 y A6, con crochets en mesoserie (Zahiri, 2012) .

\section{Arctiinae Leach, 1815}

Subfamilia grande y diversa con 11000 especies, de las cuales 6000 son Neotropicales. Se caracterizan por la presencia del órgano timbal en metatorax, productor de sonido e involucrado en la defensa y comunicación de estos lepidópteros (Scoble, 1995).

\section{Paracles Walker, 1855}

Caracterización del imago: con pubescencia densa en todo el cuerpo, de coloración opaca, pardusca, grisácea o amarillenta (Berg, 1877).

Caracterización larval: presencia de escudo protorácico dorsal con sedas y verrugas sedosas; sin traqueobranquias, con plastrón respiratorio dorsal y un complejo sistema de verrugas sedosas torácicas y abdominales (Berg, 1877).

Hábitat: cuerpos de aguas estancadas o cuerpos de agua con flujo lento como lagos, lagunas y arroyos (Berg, 1877).

Distribución: Neotrópico 


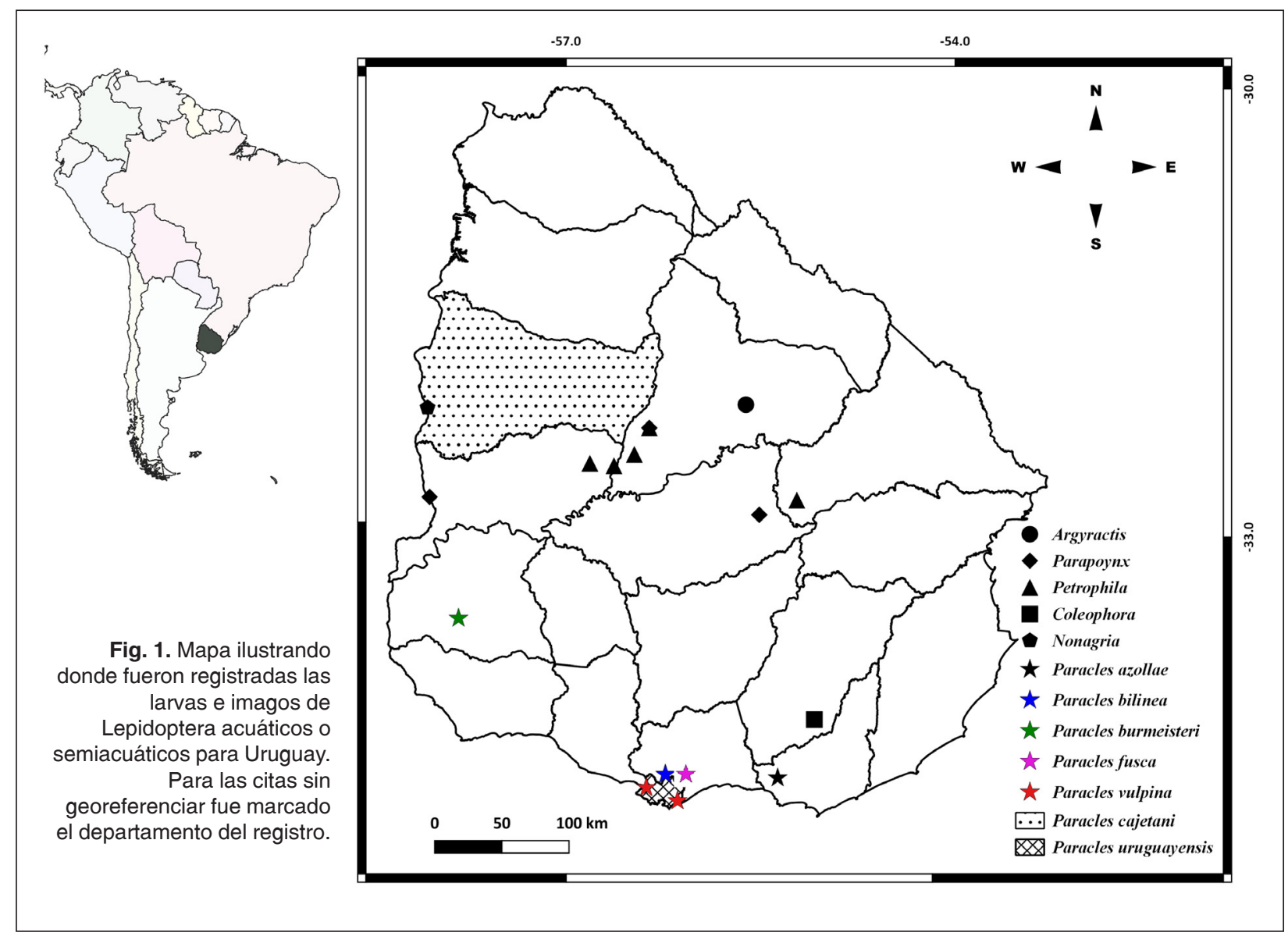

Paracles azollae (Berg, 1877)

Palustra azollae Berg, 1877

Maenas azollae (Berg, 1877)

Caracterización del imago: Expansión alar de 27$40 \mathrm{~mm}$. Hembras de mayor tamaño. Alas anteriores de color marrón oscuro, interrumpidas por máculas negras. Macho con alas posteriores amarillas, hembra con alas posteriores oscuras.

Caracterización larval: larvas del último estadio con longitud máxima de $32-35 \mathrm{~mm}$., de aspecto pubescente, erizada de sedas punzantes. Color general negro azabache, verrugas laterales rojizas, y plastrón corto dorsal de color blanco. Son larvas activas alimentándose de partes emergentes de Azolla filiculoides Lam. y de Lemna spp. Las larvas pueden sumergirse gracias a sus plastrones aeríferos. La metamorfosis ocurre en la orilla de los cursos de agua o en la superficie (Berg, 1877).

Hábitat: no hay información.

Distribución: Argentina (Buenos Aires) y Uruguay (Canelones, Maldonado).

Registro para Uruguay: VIII/2014, Sierra de las Ánimas, Maldonado (Fig. 1).

Fenología: los registros larvales para Argentina se extienden desde diciembre hasta abril (Berg, 1877).

Paracles bilinea (Schaus, 1901)

Maenas bilinea Schaus, 1901

Caracterización del imago: Expansión alar 40 mm., de color marrón oliváceo oscuro. Abdomen con banda lateral amarilla.

Caracterización larval: No hay información.

Hábitat: No hay información

Distribución: Brasil y Uruguay (Canelones)(Fig. 1).

Fenología de adultos: No hay información

\section{Paracles burmeisteri (Berg, 1877)}

Palustra burmeisteri Berg, 1877

Maenas burmeisteri (Berg, 1877)

Caracterización del imago: Expansión alar de 40$67 \mathrm{~mm}$. Hembra de mayor tamaño. Alas anteriores de color marrón oscuro, de aspecto menos escamoso que $P$. azollae. Alas posteriores oscuras y menos escamosas que las anteriores, más oscuras en vista ventral (Berg, 1877).

Caracterización larval: muy pubescente de entre 50 y $90 \mathrm{~mm}$ de longitud, de color general negro con 
reflejos verdosos. Dorso de la larva con penachos blancos dirigidos hacia atrás. Larva acuática estricta. Encontrándose en plantas sumergidas de hasta 50 $\mathrm{cm}$ de profundidad, nadan con movimientos ondulatorios. La muda se realiza en el agua, en capullos pupales adheridos a las plantas o que pueden flotar en la superficie (Berg, 1877).

Larvas se alimentan de Potamogeton pusillus L., Potamogeton crispa L., Polygonum acre K., Hydrocotyle ranunculoides L. f. (Berg, 1877).

Hábitat: No hay información

Distribución: Uruguay (Soriano) (Berg, 1877; Biezanko et al., 1978) (Fig. 1).

Fenología de adultos: No hay información

Paracles cajetani (Rothschild, 1910)

Antarctia cajetani Rothschild, 1910

Caracterización del imago: Imago con cabeza, tórax y abdomen marrón rojizo oscuro; ala anterior de color marrón rojizo con finas rayas pálidas en las venas. Ala posterior blanca (Rothschild, 1910).

Caracterización larval: No hay información.

Hábitat: No hay información

Distribución: Uruguay (Montevideo, Canelones, Paysandú) (Fig. 1).

Fenología de adultos: No hay información

\section{Paracles fusca (Walker, 1856)}

Massicyta fusca Walker, 1856;

Antarctia multifarior Burmeister, 1878

Paracles lateralis Walker, 1855

Antarctia fusca Walker, 1856

Caracterización del imago: Imago con cabeza y tórax marrón rojizo mezclados con algo de marrón grisáceo; abdomen marrón rojizo oscuro. Ala anterior pardo rojizo irradiado con marrón. Ala posterior blanca, el margen interno revestido de amarillo (Burmeister, 1878).

Caracterización larval: Larva cubierta de sedas negras, observada en hierbas de bajo crecimiento (Burmeister, 1878).

Hábitat: No hay información

Distribución: Brasil, Argentina, Uruguay (Canelones, Montevideo) (Fig. 1).

Fenología de adultos: No hay información

\section{Paracles tenuis (Berg, 1877)}

Palustra tenuis Berg, 1877

Maenas tenuis (Berg, 1877)

Caracterización del imago: Expansión alar de 36$50 \mathrm{~mm}$. Hembra de mayor tamaño que el macho. Imago con la parte anterior del cuerpo y las alas marrón amarillento. Alas posteriores oscuras. En las alas anteriores es difícil ver las bandas transversales, que tienen la misma posición y la misma dirección que en $P$. azollae (Berg, 1877).

Caracterización larval: No hay información.

Hábitat: No hay información

Distribución: Argentina (Riachuelo, Buenos Aires) y Uruguay (Silveira Guido, 1965).

Fenología de adultos: No hay información

Larvas observadas forrajeando sobre Eichhornia crassipes (Mart.) (Silveira Guido, 1965).

Paracles uruguayensis (Berg, 1886)

Palustra uruguayensis Berg, 1886

Caracterización del imago: similar a $P$. laboulbeni, pero se distingue perfectamente por las alas anteriores más estrechas, sin ápice puntiagudo y el margen costal muy redondeado, sin ángulo inferior marcado, ausencia total de líneas y manchas, alas posteriores más estrechas y su borde regularmente redondeado (Berg, 1886).

Caracterización larval: No hay información.

Hábitat: No hay información

Distribución: Uruguay (Montevideo) (Fig. 1).

Fenología de adultos: No hay información

\section{Paracles vulpina (Hübner, [1825])}

Antarctia vulpina Hübner, [1825]

Arctia pallicosta Boisduval, 1859

Caracterización del imago: machos de cabeza y tórax marrón rojizo brillante; abdomen dorsalmente anaranjado. Ala anterior marrón rojizo brillante; el margen costal blanco amarillento. Ala posterior blanca. Hembras con ala anterior con el margen costal ligeramente pálido (Hampson, 1901).

Caracterización larval: No hay información.

Hábitat: No hay información

Distribución: Argentina (Buenos Aires) y Uruguay (Canelones, Montevideo).

Registro: Humedales Santa Lucía. Montevideo.VIII2016 (Fig. 1).

Fenología de adultos: diciembre (datos personales)

\section{Crambidae (Latreille, 1810)}

Familia de amplia distribución y compuesta por 15 subfamilias con 11600 especies aproximadamente. Se caracterizan los adultos por poseer la caja timpánica abierta y el praecinctorium presente (Scoble, 1995; Romero \& Navarro, 2009).

\section{Acentropinae Stephens, 1836}

Subfamilia mejor adaptada al ambiente acuático y de amplia distribución en Sudamérica (Klima, 1937; Lange, 1956a,b; Munroe et al., 1995; Munroe \& Solis, 1999), la mayoría de las especies han sido descritas para Brasil y unas pocas para Argentina, Bolivia, Colombia, Ecuador, Guayana Francesa, Paraguay, Perú, Surinam, Venezuela y Uruguay. Agrupa adultos pequeños de hasta $30 \mathrm{~mm}$ de envergadura alar de aspecto frágil con alas de colores castaños, amarillos y dorados, con puntos negros y bordes plateados en alas posteriores. Las larvas de hábitos subacuáticos se alimentan de las plantas hospederas y pueden construir estuches de hojas portátiles o perforar túneles en brotes, tallos, pecíolos o raíces de las plantas acuáticas. Las larvas acuáticas estrictas poseen traqueobranquias 
funcionales, desarrollándose en diversos ambientes acuáticos de ríos y lagos (Romero \& Navarro, 2009).

\section{Parapoynx Hübner, [1825]}

Caracterización del imago: no sobrepasan los 30 $\mathrm{mm}$ de envergadura alar. Patrones de coloración alar castaños, amarillos, gris, blanco y dorados con puntuaciones negras sobre los bordes de las alas posteriores (Munroe \& Solis, 1999).

Caracterización larval: patrón complejo de traqueobranquias torácicas y abdominales con distintos grados de ramificaciones (Romero \& Navarro, 2009). Larvas con La ninfosis se completa dentro de brotes, tallos, peciolos o raíces donde la larva perfora túneles o dentro de larvales portátiles. Las especies de Parapoynx construyen habitáculos aglutinando trozos de hojas de las plantas hospederas con secreciones sedosas (Forbes, 1910; Kinser \& Neunzing, 1981; Buckingham \& Bennet, 2001).

Hábitat: arroyos interiores de corriente rápida de fondo de piedra con abundante vegetación de ribera y sotobosque asociado (Romero \& Navarro, 2009), de hábitos semiacuáticos y acuáticos siempre asociadas a plantas acuáticas sumergidas o emergentes (Habeck, 1974; Herlong, 1979).

Distribución: México, Honduras, Surinam, Colombia, Brasil, Argentina y Uruguay (Río Negro, Tacuarembó). Registros: V- 2002. Ao San Ramón Chico (Esteros de Farrapos, Río Negro); Ruta 20 (Departamento Río Negro); Afluente $A \square$ Tres árboles (Departamento Río Negro); dos $A \square$ afluentes de Salsipuedes (Departamento Tacuarembó) (Fig. 1).

Fenología de adultos: No hay información

\section{Petrophila Guilding, 1830}

Caracterización del imago: de aspecto muy escamoso.

Caracterización larval: procoxas separadas; patrón de traqueobranquias torácicas y abdominales filamentosas simples, supra y subespiraculares (Romero \& Navarro, 2009).

Hábitat: Larvas sedentarias asociadas a sustratos de ríos libres de contaminación y alto contenido de oxígeno (Romero \& Navarro, 2009).

Distribución: México, Guayana Francesa, Surinam,Venezuela, Colombia, Perú, Ecuador, Brasil, Argentina, Uruguay (Cerro Largo, Durazno y Tacuarembó).

Registro: IV- 2015. Ao Los Molles (Cerro Largo); $A \square$ afluente de Salsipuedes (Departamento Tacuarembó); Durazno (Fig. 1).

Fenología de adultos: No hay información

\section{Argyractis Hampson, 1894}

Caracterización del imago: pequeños, frágiles a la vista. Patrones variados de coloración alar con alas doradas y puntos negros.

Caracterización larval: procoxas fusionadas; patrón sedoso torácico y abdominal con traqueobranquias filamentosas simples con distintos números de agrupación (Romero \& Navarro, 2009).

Hábitat: Se ha descrito la construcción de refugios larvales utilizando secreciones sedosas para entretejer raíces de plantas acuáticas (Romero \& Navarro, 2009).

Distribución: Guayanas, Brasil, Argentina, Uruguay (Tacuarembó) Registro: VI- 2015/2016. Ao Claudina, afluente del río Tacuarembó (Tacuarembó)-(Fig. 1).

Fenología de adultos: No hay información.

\section{Spilomelinae Guenée, 1854}

Subfamilia con 4000 especies, es la más diversa dentro de Crambidae. La expansión alar varía entre $11 \mathrm{~mm}$ y $50 \mathrm{~mm}$. En reposo los imagos tienen una característica forma triangular característica, con las alas generalmente sobre el abdomen, donde las alas delanteras cubren las posteriores (Romero \& Navarro, 2009).

\section{Samea Guenée, 1854}

Caracterización del imago: Abdomen muy largo, muy ahusado. Alas oblongas, lisas, brillantes, semitransparentes, con bordes opacos y flecos más o menos intercalados: las superiores estrechas, con ápice agudo y alargado.

Caracterización larval:No hay información

Hábitat: No hay información

Distribución: Colombia, Brasil, Argentina y Uruguay

(Deloach et al., 1979).

Fenología de adultos: No hay información

Samea multiplicalis (Guenée, 1854)

Isopteryx multiplicalis Guenée, 1854

Samea discessalis Walker, [1866]

Lepyrodes nicaeusalis Walker, 1859.

Caracterización del imago: de color canela con marcas más oscuras en ambos pares de alas y una envergadura de unos $20 \mathrm{~mm}$.

Caracterización larval: de color blanquecino o amarillo pálido durante los estadios tempranos y desarrollan tonalidad amarillo verdoso a medida que maduran. Larva se alimenta de Pistia stratiodes L. También acepta Eichhornia crassipes, Salvinia spp., Azollae carolineana (Willd) (Deloach et al., 1979; Julien et al., 2012).

Hábitat: Vive sobre plantas acuáticas, prospera en climas tropicales, con un crecimiento y una dispersión más lentos en climas más templados.

Distribución: Panamá, Trinidad \& Tobago, Guayanas, Brasil, Argentina y Uruguay (Deloach et al., 1979).

Fenología de adultos: no hay información

\section{Pyralidae (Latreille, 1802)}

Compuesta por 5000 especies en 5 subfamilias, para el Neotrópico sólo cuenta con representantes con hábitos semiacuáticos dentro de la subfamilia Phycitinae. Las larvas acuáticas de esta subfamilia 
son barrenadoras o perforadoras de tallos, distinguiéndose por el área esclerotizada en el mesotórax (Romero \& Navarro, 2009).

\section{Nomophila indistinctalis (Walker, 1863)}

Nephopteryx indistinctalis Walker, 1863

Nomophila noctuella (Denis \& Schiffermüller, 1775)

Caracterización larval: No hay información

Hábitat: Larva acuática barrenadora de lagunilla

(Alternathera philoxeroides).

Distribución: Brasil y Uruguay (Munroe, 1995).

Fenología de adultos: No hay información

\section{Pyraustinae Meyrick, 1890}

Amplia distribución (Munroe \& Solis, 1999), adultos con alas anteriores triangulares y penachos escamosos en el área discal. Larvas semiacuáticas carentes de branquias, con espiráculos funcionales. Son larvas generalistas alimentándose de hojas y tallos de las plantas hospederas.

\section{Neohelvibotys pelotasalis (Capps, 1967)}

Loxostege pelotasalis Capps, 1967

Caracterización del imago: Expansión alar de 18$20 \mathrm{~mm}$.

Caracterización larval: No hay información

Hábitat: Larva acuática barrenadora de lagunilla

(Alternathera philoxeroides)

Distribución: Uruguay y Brasil (Munroe, 1995)

Fenología de adultos: mayo.

\section{Niphograpta albiguttalis (Warren, 1889)}

Epichronistis albiguttalis Warren, 1889

Sameodes albiguttalis (Warren, 1889)

Caracterización del imago: ala anterior de color amarillo dorado moteado de gris, y con sombreado gris y con conspicuas manchas blancas a lo largo; mancha discal blanca, cuadrada, bordeada arriba y abajo por una mancha con borde negro; alas posteriores de color amarillo brillante con una mancha discal negra prominente; abdomen marrón rojizo con banda blanca en la base de cada segmento (Munroe, 1995).

Caracterización larval: larva de último estadio con cabeza marrón oscura anaranjada, largo máximo de $18 \mathrm{~mm}$, de hábitos minadoras se alimenta de Eichhornia crassipes, Salvinia spp y Azolla filiculoides Lan (Center et al., 1982). Controladora de camalote $E$. crassipes (Julien et al., 2012).

Hábitat: Cuerpo de agua, con plantas acuáticas, larvas se alimentan de Eichhornia crassipes.

Distribución: Brasil, Argentina y Uruguay (SilveiraGuido, 1965)

Fenología de adultos: No hay información.

\section{Noctuidae Latreille, 1809}

Aunque es una familia de gran diversidad, tiene pocas especies con hábitos acuáticos en Sudamérica. Las larvas de las especies acuáticas de estas familias son minadores de hojas y tallos durante los primeros estadios, característica que las vuelve atractivas para el control de camalote como Erichhornia cassipes (Romero \& Navarro, 2009).

Nonagria Oschsenheiner, 1816

Caracterización del imago: Cuerpo de colores oscuros, ala posterior con venas Subcostal y Radial fusionadas por una corta distancia en la base de la celda discal; abdomen sin marcas lateromedianas (Romero \& Navarro, 2009).

Caracterización larval: larva desnuda, ausencia de traqueobranquias y plastrón, espuripedios con crochets uniordinales. Primeros estadios larvales con hábitos minadores (Romero \& Navarro, 2009).

Hábitat: arroyos interiores de corriente rápida de fondo de piedra con abundante vegetación de ribera y sotobosque asociado (Romero \& Navarro, 2009).

Distribución: Ecuador, Brasil, Uruguay (Paysandú) Registros: IX- 2007. Ao San Francisco (Paysandú) (Fig. 1)

Fenología de adultos: sin información.

\section{Coleophoridae Bruand, 1850}

Microlepidopteros cosmopolitas de pequeños a mediana envergadura, de alas angostas. Larvas con placas dorsales bien desarrolladas, las larvas de especies de hábitos acuáticos frecuentan la vegetación marginal emergente. Son en general larvas minadoras o perforadoras de tallos, con la característica de construir refugios de seda y partes de las plantas hospederas. Estos habitáculos ya pueden ser construidos por larvas de primer estadio o en los últimos estadios donde ocurrirá la muda pupal. Fijan capullos a la planta hospedera. Los mismos son bastantes característicos de cada especie.

\section{Coleophora Hübner, 1822}

Coleophora contiene cerca de 1000 especies descritas, pero pocas registradas para el Neotrópico. Actualmente se conocen 17 especies, de las cuales dos son exóticas, siete especies para Argentina, una para Brasil, entre otros (Pastrana, 1963; Baldizzone, 2020).

Caracterización del imago: Ala anterior acuminada, ala posterior lineal lanceolada; ala anterior de colores opacos; tergitos abdominales con manchones espinosos (Romero y Navarro, 2009)

Caracterización larval: orugas con patas torácicas presentes; tórax y abdomen sin sin filamentos branquiales; larvas constructoras de habitáculos portátiles. Espuripedios con crochets en bandas transversales uniseriales; tórax con placas dorsales bien desarrolladas (Romero \& Navarro, 2009).

Hábitat: son individuos de hábitos minadores de vegetación marginal emergente (semiacuáticos), característicos por construir habitáculos de material vegetal (Romero \& Navarro, 2009). 
Distribución: Argentina, Brasil, Perú, Chile y Uruguay (Lavalleja). Registro: IX- 2019. Ao La Tigresa, Villa Serrana (Lavalleja)-(Fig. 1).

Fenología de adultos: no hay información.

\section{DISCUSIÓN}

De acuerdo a los trabajos de Mey \& Speidel (2008) y Romero \& Navarro (2009) se citan para América del Sur ocho familias con estados inmaduros asociados ambientes dulceacuícolas, mientras en Uruguay hemos registrados cinco; se mencionan 36 géneros, mientras son 10 los citados para Uruguay. Brasil cuenta con 24 géneros citados seguido por Argentina con 13 y México con 10 al igual que Uruguay, por debajo se distribuirán el resto de los países. Por otra parte, el género con más especies asociadas a ambientes acuáticos es Paracles, la mayor riqueza específica la presenta Brasil (27 spp.), seguido por Argentina (16 spp.), Colombia (10 spp.) y luego Uruguay con ocho especies. En el presente estudio se identificaron para Uruguay cinco familias: Erebidae, Crambidae, Pyralidae, Noctuidae y Coleophoridae; 10 géneros: Paracles, Parapoynx, Petrophila, Argyractis, Samea, Nomophila, Neohelvibotys, Niphograpta, Nonagria y Coleophora. Y un total de 12 especies distribuidas de la siguiente forma: Paracles con ocho especies ( $P$. azollae, $P$. bilinea, $P$. burmeisteri, $P$. cajetani, $P$. fusca, $P$. tenuis, uruguayensis, $P$. vulpina), Samea multiplicalis, Nomophila indistinctalis, Neohelvibotys pelotasalis, Niphograpta albiguttalis. y seis especies que no se han logrado identificar ( por ser larvas) de varios géneros (Parapoynx, Petrophila, Argyractis, Nomophila, Nonagria y Coleophora). Se registra la larva del género Coleophora por primera vez para Uruguay, con material proveniente del departamento de Lavalleja. De igual forma también este trabajo aporta los lugares de muestreo donde se han encontrado estas larvas, esto permite visualizar los vacíos de información, debido a la falta de un relevamiento sistemático de este tipo de fauna. Esta información podría orientarnos en la localización de los hábitats de preferencia sin ser excluyentes ya que no se cuentan con trabajos de síntesis a nivel de géneros ni de especies. A medida que se realicen un mayor esfuerzo de muestreo para el resto del país, así como la identificación del material que en la mayoría de los casos es desechado en los muestreos de macroinvertebrados acuáticos será posible incorporar la lepidopterofauna acuática en los programas de monitoreo ambiental, evaluación ecosistémica e incorporar estas familias dentro del espectro de bioindicadores para calidad de agua. Son de gran importancia los inventarios de artropodofauna asociada a ecosistemas de agua dulce para futuros estudios de biología, ecología, y diversidad.

\section{BIBLIOGRAFÍA}

Baldizzone G. 2020. The South American Coleophoridae of the Zoological Museum of Copenhagen. Contribution to the knowledge of the Coleophoridae. CXLIII (Lepidoptera: Coleophoridae). SHILAP Revista de lepidopterología, 48 (190): 197-222.

Beccacece H., Vincent B. \& F.R. Navarro. 2014. The type-material of Arctiinae (Lepidoptera, Erebidae) described by Burmeister and Berg in the collection of the Museo Argentino de Ciencias Naturales Bernardino Rivadavia (Buenos Aires, Argentina). ZooKeys, 421: 65-89.

Bentancur-Viglione M.G. \& E. Morelli. 2012. Primer registro de Parapoynx (Forbes, 1910) para el Uruguay (Lepidoptera: Crambidae). IV Encuentro de Lepidoptera Neotropicales, Montevideo.

Bentancur-Viglione M.G. \& E. Morelli. 2014. Aportes a la taxonomía larval del género Parapoynx (Lepidoptera: Crambidae). IV Congreso Colombiano Zoología, Cartagena de Indias.

Bentancur-Viglione M.G. \& E. Morelli. 2015. Patrón sedo-branquial en la taxonomía larval del género Parapoynx Hübner, 1825 (Lepidoptera: Crambidae). Memorias del X Convención Internacional sobre Medio Ambiente $y$ Desarrollo. DOI: 10.13140/RG.2.2.20075.13602

Bentancur-Viglione M.G., Arocena R., Castro M., Vernassa T. \& E. Morelli. 2016.

Primer registro del género Petrophila (Whidding, 1830) para Uruguay (Lepidoptera: Crambidae), aportes a la taxonomía larval. IV Congreso Uruguayo de Zoología. DOI: 10.13140/RG.2.2.18338.43204

Berg C. $1876 a$. Memorias sobre orugas acuáticas de la familia de los Bombycidae. Annales de la Sociedad Científica Argentina, 2: 184-190.

Berg C. 1876b. Palustra Azollae y Palustra tenuis. (Memorias leída en la asamblea del 15 de septiembre de 1876). Annales de la Sociedad Científica Argentina, 2: 241-246.

Berg C. 1877. Estudios Lepidopterológicos acerca de la fauna Argentina y Oriental. Annales de la Sociedad Científica Argentina, 3: 228:234.

Berg C. 1886. Une nouvelle espèce de Bombycide. Bulletin entomologique. Annales de la Societe Entomologique de France, (6) 5: ccxii-ccxiii [212213]

Biezanko C.M., Ruffinelli A. \& D. Link. 1978. Catálogo de lepidópteros do Uruguai. Revista Centro Ciências Rurais, 4: 107-148.

Buckingham G.R \& C.A. Bennet. 1996- Laboratory biology of an immigrant asian moth Parapoynx diminutalis (Lepidoptera: Pyralidae), on Hydrilla verticillata (Hydrocharitaceae). Florida Entomologist, 79: 353-363.

Buckingham G.R \& C.A. Bennet. 2001- Life history and laboratory host range test of Parapoynx 
seminealis (Walker) (Crambidae: Nymphulinae) in Florida, USA. Journal of the Lepidopterists' Society, 55: 111-118.

Burmeister 1878. Description physique de la République Argentine d'après des observations personelles et étrangeres. Vol.5. Lépidoptères. Première partie. Contenant les diurnes, crépusculaires et bombycoïdes. Avec un Atlas de XXIV Planches In-4e. 526p.

Carter D.J. \& N.P. Kristensen. 1998. Classification and Keys to higher taxa. pp 27-40. En: Kristensen, N. P. (ed) Lepidoptera, Moth and Butterflies, Volumen 1: Evolution, Systematics, and biogeography. Handbuch der Zoologie Band IV Arthropoda: Insecta. Teilband / part-35. Walter de Gruyter. Berlin. New York. 491 p.

Center T.D., Balciunas J.K. \& D.H. Habeck. 1982. Descriptions of Sameodes albiguttalis (Lepidoptera: Pyralidae) life stages with key to Lepidoptera larvae on waterhyacinth. Annals of the Entomological Society of America, 75(4): 471-479.

DeLoach C.J., DeLoach A.D. \& H.A. Cordo. 1979. Observations on the biology of Samea multiplicalis on waterlettuce in Argentina. Journal of Aquatic Plant Management, 17: 42-44.

Domínguez E. \& H.R. Fernandez. 2001. Guía para la determinación de los artrópodos bentónicos sudamericanos, Serie: Investigaciones de la UNIT, Subserie: Ciencias Exactas y Naturales Universidad Nacional de Tucumán. Editorial Universitaria de Tucumán. 287 p.

Drechsel U. 2014. Aquatic habit of larval instar of Paracles palustris (Joergensen, 1935) (Lepidoptera: Erebidae: Arctiinae). Paraguay Biodiversidad, 1(18): 89-94.

Drechsel U. \& S. Drechsel García. 2016a. The immature stages of Paracles contraria Walker, 1855 (Lepidoptera: Erebidae: Arctiinae). Paraguay Biodiversidad, 3(6): 33-38.

Drechsel U. \& S. Drechsel García. 2016b. The early stages of Paracles fusca (Walker, 1856) (Lepidoptera: Erebidae: Arctiini). Paraguay Biodiversidad, 3(8): 47-52.

Drechsel U. \& S. Drechsel García. 2017. The early stages of Paracles aurantiaca (Rothschild, 1910) (Lepidoptera: Erebidae: Arctiini) Paraguay Biodiversidad, 4(1): 1-7.

Forbes W.T.M. 1910. The aquatic caterpillars of lake Quinsigamond. Psyche, 27: 219-227.

Habeck D.H. 1974. Caterpillars of Parapoynx in relation to aquatic plants in Florida. Hyacinth Control Journal, 12: 15-18.

Habeck D.H. \& S.C. Passoa. 1987. A description of the larva and pupa of Rupela albinella, a pest of rice in Latin America (Lepidoptera: Pyralidae: Schoenobiinae). The Florida Entomology, 7(3): 368-375.

Hampson G.F. 1901. Catalogue of the Arctiidae
(Arctiinae) and Agaristidae in the collection of the British Museum (Nat. Hist.) Catalogue of the Lepidoptera Phalaenae in the British Museum. 3: i-xix, 1-690, pl. 36-54

Herlong D.D. 1979. Aquatic Pyralidae (Lepidoptera: Nymphulinae) in South Carolina. The Florida Entomologist, 62 (3): 189:193.

Hinton H.E. 1946. On the homology and nomenclature of the setae of Lepidopterous larvae, with some notes on the phylogeny of the Lepidoptera. Transaction of the Royal Entomological Society of London, 97: 1-37.

Julien M.H., McFadyen R.E. \& J. Cullen. 2012. Biological Control of Weeds in Australia. Csiro Publishing, $620 \mathrm{p}$.

Kinser P.D. \& H.H. Neunzing. 1981. Description of the immature stages and biology of Synclita tinealis Munroe (Lepidoptera: Pyralidae: Nymphulinae). Journal of the Lepidoptery Societe, 35: 137-146.

Klima A. 1937. Pyralidae: Subfam.: Scopariinae et Nymphulinae. En: Bryk F, editor. Lepidopterorum Catalogus, 84: 1-226

Lange W. H. 1956a. A generic revision of the aquatic moth of North America; (Lepidoptera: Pyralidae, Nymphulinae). Wasmann Journal Biology, 14 (1): 59-144.

Lange W. H. 1956b. Cap 11. Aquatic Lepidoptera. 271-292. pp En: Usinger , R.L. ed. Aquatic insects of California. University of California. Editorial Berkeley and Los Angeles.

Lange W. H. 1978. Aquatic and Semiaquatic Lepidoptera, 187- 201. En: Merrit R. W. and K. W. Cummins (eds). An introduction to the Aquatic Insect of North America. Kendall/ Hunt Publishing Company. $1158 \mathrm{p}$.

MacGaha Y. J. 1954. Contribution to the biology of some Lepidoptera which feed on certain aquatic flowering plants. Transactions of the American Microscopical Society, 73: 167-177.

Maddox D. M. 1970. The bionomics of a stem borer, Voctia malloi (Lepidotera: Phycitinae) on alligator weed in Argentina. Annals of the Entomological Society of America 63(5): 1267-1273.

Meneses A. R., Bevilaqua M. V. O., Hamada N. \& R. B. Querino. 2013. The aquatic habitat and host plants of Paracles klagesi (Rothschild) (Lepidoptera, Erebidae, Arctiinae) in Brazil. Revista Brasileira de Entomologia, 57(3): 350-352.

Mey W. \& W. Speidel. 2008. Global Diversity of butterflies (Lepidoptera) in freshwater. Freshwater Animal Diversity Assessment. Hydrobiologia, 595: 521-528.

Morelli E. \& M.-G. Bentancur-Viglione. 2012. Primer registro de Nonagria (Insecta Lepidoptera) para el Uruguay. Primer congreso latinoamericano de macroinvertebrados acuáticos, San José de Costa Rica, 2012

Morelli E. \& M.-G. Bentancur-Viglione. 2013. Lepidopterofauna Acuática de Uruguay. Folia 
Entomológica Acta Primer congreso latinoamerica. 1536-1539.

Morelli E., Arocena R., Castro M. \& M. G. BentancurViglione. 2018a. Primer registro del género Argyractis Hampson, 1894 en ecosistemas dulceacuícolas de Uruguay (Lepidoptera: Crambidae, Nymphulinae). Congreso Aquatrop, Quito, Ecuador.

Morelli E., Díaz A. \& M. G. Bentancur-Viglione. 2018b. Descripción de la larva de

Paracles azollae (Berg, 1877) (Lepidoptera: Erebidae). Boletín de la Sociedad

Zoológica del Uruguay, 27 (2): 34-40.

Munroe E. G. 1974. Three new genera and four new species of Pyralidae (Lepidoptera) from South America. Canadian Entomology, 106: 11-20.

Munroe E. G. 1995. Crambidae, pp. 34-79. En: Heppner, J. B. (ed.) Atlas of Neotropical Lepidoptera. Checklist part 2; Pyraloidea to Totricoidea, Association for Tropical Lepidoptera. Scientific Publishers, Gainesville, Florida, USA. 287 p.

Munroe E. G., Becker V. O., Shaffer J. \& M. A. Solis. 1995. Pyralidae, pp. 80-105. En: Heppner, J. B (ed). Checklist Part 2: Pyraloidea to Tortricoidea. Atlas of Neotropical Lepidoptera. Gainesville: Association for Neotropical Lepidoptera. 287 p.

Munroe E. G. \& M. A. Solis. 1999. Pyraloidea, pp 233256. En: Kristensen, N. (ed). Lepidoptera, Moths and Butterflies, Vol. 1, Arthropoda, Insect, Vol. 4, Part 35. Handbook of Zoology. Walter de Gruyter \& Co. Berlin. 491 p.

Nessimian J. L \& E. R. Da Silva. 1997- Descrição das formas imaturas da Parapoynx restingalis (Da Silva \& Nessimian, 1990) (Pyralidae: Nymphulinae), com notas biológicas. Revista Brasileira de Biología, 54: 600-610.

Pastrana J. A. 1963- La familia Coleophoridae (Lepidoptera) en América Latina. Revista de la Sociedad Entomológica Argentina, 26: 89-101.

Roback S. S. 1966. The Catherwood foundation
Peruvian-Amazon expedition 10. Aquatic Lepidoptera (Pyralidae, Nymphulinae). Monographs - Academy of Natural Sciences of Philadelphia, 225-233.

Romero V. F. \& F. Navarro. 2009. Lepidóptera, 309340. En: Dominguez \& Hernández (eds) macroinvertebrados bentónicos sudamericanos (primera ed.). San Miguel de TucumánArgentina, Fundacion Miguel Lillo. 656p.

Rothschild. 1910. Descriptions of new species of Arctianae in the Tring Museum Novitates Zoologicae, 17 (2): 172-188.

Sattler W. 1961. Ein in de mertamorphoseestadden aquatisches polyrheobiontes lepidopter (Elophila, Pyralidae) aus de Tumucumaque Bergland (Brasilianisch, Guayana) Bol. Mus. Par., 36: 1-11.

Silveira Guido A. 1965. Natural enemies of weed plants. Final report on PL-480. Project S9-CR1 (Jan 1962 to 15 Nov. 1965). Universidad de la República, Facultad de Agronomía, Montevideo, Uruguay.

Stehr F.-W. 1987. Order Lepidoptera, p.288-315. En: F.-W. Stehr (ed.), Immature insects. v. I, Kendall/ Hunt Publishing Company, Dubuqueque, 754p.

Tuskes P. M. 1977. Observations on the biology of Parargryactis confusalis an acuatice Pyralid (Lepidoptera: Pyralidae). Canadian Entomology, 109: 695-699.

Welch P. S. 1922. The respiratory mechanism in certain aquatic Lepidoptera. Transactions of the American Microscopical Society, 41: 29-50.

Zahiri R. 2012. Molecular phylogenetics of Erebidae (Lepidoptera, Noctuoidea). Systematic Entomology 37: 102-124. DOI:10.1111/j.13653113.2011.00607.x.

Fecha de Recepción: 09 de noviembre de 2020 Fecha de Aceptación: 17 de diciembre 2020 